\title{
Effect of five enological practices and of the general phenolic composition on fermentation-related aroma compounds in Mencia young red wines
}

\author{
Ana Añón , Jorge F. López , Diego Hernando , Ignacio Orriols , Eugenio Revilla ，Manuel M. Losada
}

\begin{abstract}
A B S T R A C T
The effects of five technological procedures and of the contents of total anthocyanins and condensed tannins on 19 fermentation-related aroma compounds of young red Mencia wines were studied. Multifactor ANOVA revealed that levels of those volatiles changed significantly over the length of storage in bottles and, to a lesser extent, due to other technological factors considered; total anthocyanins and condensed tannins also changed significantly as a result of the five practices assayed. Five aroma compounds possessed an odour activity value $>1$ in all wines, and another four in some wines. Linear correlation among volatile compounds and general phenolic composition revealed that total anthocyanins were highly related to 14 different aroma compounds. Multifactor ANOVA, considering the content of total anthocyanins as a sixth random factor, revealed that this parameter affected significantly the contents of ethyl lactate, ethyl isovalerate, 1-pentanol and ethyl octanoate. Thus, the aroma of young red Mencia wines may be affected by levels of total anthocyanins.
\end{abstract}

\section{Introduction}

Volatile compounds released during alcoholic fermentation are the main aroma components of young red wines (Rapp \& Mandery, 1986). These aroma components consist of dozens of alcohols, organic acids, esters, carbonyl compounds, sulfur compounds, nitrogen compounds, lactones and volatile phenols. Several authors have demonstrated that many factors (e.g., grape amino acids composition, sugar content and $\mathrm{pH}$ of must, type of yeast, temperature and aeration during the alcoholic fermentation, and length of maceration) have remarkable effects on the concentration of aroma compounds in wines (Callejon et al., 2010; Perestrelo, Fernandes, Alburquerque, Marques, \& Camara, 2006). Furthermore, cold prefermentative maceration yields young red wines with higher contents of acetates and other esters than conventional winemaking does (Alvarez, Aleixandre, Garcia, \& Lizama, 2005).

It is well known that several post-fermentation practices may change the characteristics of red wines made with similar sets of grapes. Changes in the aroma composition of red wines aged in oak casks are more pronounced if compared with wines stored in stainless steel tanks. First of all, the contact of wine with oak casks gives rise to a higher concentration of aroma compounds extracted from oak wood (Chatonnet, Boidron, \& Pons, 1990); on the other hand, some aroma compounds formed during alcoholic fermentation are absorbed by oak wood, lowering their levels in wines (Ramirez et al., 2001).

During the storage of wine in bottles, several chemical changes in volatile composition, like hydrolysis and formation of esters and acetals, take place. This is owing to a series of reactions that depend on the composition of wine (concentration of ethanol, $\mathrm{pH}$ ), the time the wine is stored in the bottle and the storage temperature (Ramey \& Ough, 1980; Rapp \& Mandery, 1986). The effect of temperature during the storage of red wines in bottles has been studied by several authors (Puech, Vidal, Pegaz, Riou, \& Vuchot, 2006); sensory and chemical changes were less intense when wines were stored at low temperatures. These data suggest that the control of temperature during the storage of wines in bottles may be used to extend the shelf-life of young red wines. On the other hand, it is widely accepted that relatively high temperatures and low $\mathrm{pH}$ increase the hydrolysis of acetates and ethyl esters during wine storage (Ramey \& Ough, 1980) and, as a consequence, the intensity of fruity aromas decreases. 
Colloidal macromolecules of wines may interact with aroma compounds, as has been proved for mannoproteins released by yeast (Lubbers, Charpentier, Feuillat, \& Voilley, 1994). Interactions between macromolecules and aroma compounds may be repulsive, increasing the volatility of aroma compounds; but may also be attractive, leading to the retention of aroma compounds by colloids. Of course, those interactions depend on the nature of macromolecules and on the type of considered aroma compound (Chalier, Angot, Delteil, Thierry, \& Gunata, 2007). The protective effect of anthocyanins extracts and malvidin-3-O-glucoside on volatile thiols in model solutions during maturation has been demonstrated (Murat, Tominaga, Saucier, Glories, \& Dubourdieu, 2003), and these authors have suggested that the effect may be related to the antioxidant properties of anthocyanins.

Mencia grapes are widely cultivated in north-western Spain. Some studies on the phenolic and aroma composition of red wines made with Mencia grapes have been carried out. They have focused on the characterisation of the aroma profile of Mencia wines (Calleja \& Falque, 2005; Noguerol-Pato, Gonzalez-Barreiro, Cancho-Grande, \& Simal-Gandara, 2009), on changes of aroma profile of Mencia wines caused by the geographic origin of grapes and by the characteristics of each year (Vilanova et al., 2012), and on modifications of the phenolic composition of those wines during their storage in bottle (Garcia-Falcon, Perez-Lamela, Martinez-Carballo, \& Simal-Gandara, 2007) or caused by the use of maceration enzymes and cold prefermentative maceration (Ortega-Heras, Perez-Magarino, \& Gonzalez-Sanjose, 2012). Nevertheless, these studies have not considered the effect of other oenological procedures, like winemaking technology, maturation in oak barrels or storage in stainless steel tanks, and the characteristics of storage in bottles on the aroma profile and on the general phenolic composition of Mencia wines. Moreover, the interactions among different technological factors have not been studied, both in Mencia and in other types of wines. For these reasons, a study on the effect of several technological factors on the aroma and general phenolic composition of Mencia wines has been carried out. In addition, the interactions among general phenolic composition and aroma components have been considered.

\section{Materials and methods}

\subsection{Reagents, standards and enological products}

All reagents, analytical grade, were supplied by Panreac (Barcelona, Spain). Analytical standards for GC-MS analysis were obtained from Sigma-Aldrich Quimica (Tres Cantos, Spain). Gelatine and sodium bentonite were supplied by Agrovin (Alcázar de San Juan, Spain). Sulfur dioxide was provided by AirLiquide Espafia (Madrid, Spain).

\subsection{Grapes and winemaking}

Mencia grapes were harvested during the month of September in a 15-year-old vineyard, located $(42.25 \mathrm{~N}, 6.59 \mathrm{~W})$ in the area of Appellation d'Origine Contrôlé Valdeorras, at about $400 \mathrm{~m}$ a.s.l., and close to the Sil river, in an environment characterised by high relative humidity (about $80 \%$ ) during the month of September. Plants, grafted on 110 Richter and trained on Cordon Royat system, are arranged $\mathrm{N}-\mathrm{S}$ on a $<5 \%$ slope, vine spacing being $2.50 \mathrm{~m} \mathrm{x}$ $1.40 \mathrm{~m}$. Harvest took place by the end of September 2005. Grapes $(5000 \mathrm{~kg})$ were hand-harvested, placed in $20-\mathrm{kg}$ plastic boxes, and transported to the winery. Winemaking was carried out in the Jesus Nazareno Cooperative Cellar (El Barco de Valdeorras, Orense, Spain). Grapes were crushed and de-stemmed, and were later treated with sulfur dioxide $\left(50 \mathrm{mg} \mathrm{\textrm {L } ^ { \prime 1 }}\right)$.
Two 2500-L stainless steel tanks were used for winemaking. In one tank, a traditional winemaking was carried out. In the other tank, a prefermentative cryomaceration was carried out, using dry ice to drop the temperature to $4-5{ }^{\circ} \mathrm{C}$; this temperature was maintained for three days, adding dry ice when necessary. In both cases, tanks were inoculated with $40 \mathrm{~g} / 100 \mathrm{~L}$ Levuline BRG yeast (Lallemand, Alcázar de San Juan, Spain); temperature was maintained at $26{ }^{\circ} \mathrm{C}$ during the alcoholic fermentation, and must was pumped over twice a day. At the end of alcoholic fermentation, wines were transferred to another stainless steel tank, and Uvaferm Alpha lactic bacteria (Lallemand, Alcázar de San Juan, Spain) were inoculated, following the manufacturer's instructions, to ensure an optimal development of malolactic fermentation. Then, wines were transferred to other stainless steel tanks. The level of sulfur dioxide was adjusted to $30 \mathrm{mg} \mathrm{L}$, , remaining in those tanks until spontaneous sedimentation of lees took place. Thus, two different young red wines were obtained: a control wine made with traditional winemaking (T), and a wine made after cold prefermentative maceration $(\mathrm{F})$.

\subsection{Maturation and storage of wines}

In February 2006, each type of wine was submitted to two different maturation practices, using two types of containers: 400-L stainless steel tanks (A) and 225-L middle-toasted American oak barrels (R); barrels were purchased from Magreñann (Logroño, Spain). For each wine, two stainless steel tanks and two oak barrels were used; wines were maintained in the maturation containers for two or three months. Thus, eight different wines were obtained after the maturation process. After maturation, wines were treated with gelatine $(2 \mathrm{~g} / 100 \mathrm{~L})$ and sodium bentonite $(30 \mathrm{~g} / 100 \mathrm{~L})$, filtered through diatomaceous earth, stabilised at $-4{ }^{\circ} \mathrm{C}$ for 10 days, and finally filtered through a filter press. Then wines were bottled in 750-mL glass bottles, using premium cork stoppers. For each wine, two different sets of bottles were obtained. A set of bottles was maintained in a chamber at $14{ }^{\circ} \mathrm{C}$ (set C). The other set remained at room temperature, between 10 and $30^{\circ} \mathrm{C}$ (set $\mathrm{V}$ ). Wines were analysed three and nine months after bottling. In summary, 32 different wines were obtained, combining five different technological factors:

$\mathrm{Fl}$ : type of winemaking ( $\mathrm{T}$ or $\mathrm{F}$ ).

F2: type of container used for maturation (A or R).

F3: length of maturation (2 or 3 months).

F4: temperature of storage in bottles $(\mathrm{C}$ or $\mathrm{V})$.

F5: length of storage in bottles (3 or 9 months).

Thus, each wine was identified by a five alpha-numeric code, e.g., wine $\mathrm{TA} 2 \mathrm{C} 3$ was obtained by traditional winemaking, matured in a stainless steel tank for two months, and stored in bottle at controlled conditions for three months.

\subsection{General analysis of must and wines}

Sugar content, $\mathrm{pH}$, total acidity and content of tartaric and malic acids in the must were analysed following OIV methods (OIV, 2010). Alcoholic degree, $\mathrm{pH}$, total acidity, volatile acidity, malic acid, reducing sugars, dry extract, total sulfur dioxide and free sulfur dioxide in wines after three and nine months of storage in bottles were determined by OIV methods (OIV, 2010).

\subsection{Analysis of volatile compounds}

Volatile compounds were determined by GC-MS in Estación Enológica de Haro (Spain), three months and nine months after bottling, following the procedure described by Prieto et al. 
(2012). Extraction of volatile compounds was carried out by direct headspace trap technique, using a Turbomatrix 40 Trap (Perkin Elmer, Waltham, MA), the vial being pressurised for $1 \mathrm{~min}$, and charged for $1.5 \mathrm{~min}$. The following temperatures were used: needle, $110{ }^{\circ} \mathrm{C}$; oven, $85{ }^{\circ} \mathrm{C}$; transfer line, $140{ }^{\circ} \mathrm{C}$; trap, $45^{\circ} \mathrm{C}$ (low) and $290^{\circ} \mathrm{C}$ (high). Pressures were as follows: vial, $30 \mathrm{psi}$; in column, $30 \mathrm{psi}$; desorption, $30 \mathrm{psi}$. Times stipulated for the different processes that take place in the headspace were: dry purge, $10.0 \mathrm{~min}$; trap at maximum temperature, $12.0 \mathrm{~min}$; desorption, $10.0 \mathrm{~min}$; thermostatisation, $45 \mathrm{~min}$. Each cycle took $108.0 \mathrm{~min}$ for each chromatographic analysis. The chromatographic separation was carried out in a Clarus 500 gas chromatograph (Perkin Elmer) using a TRB-FFAP column (60 m x $0.25 \mathrm{~mm} \times 0.25 \mathrm{urn}$ ), using the following oven temperature program: $3 \mathrm{~min}$ at $40^{\circ} \mathrm{C}$, from $40{ }^{\circ} \mathrm{C}$ to $80{ }^{\circ} \mathrm{C}$ at $2{ }^{\circ} \mathrm{C} / \mathrm{min}, 15 \mathrm{~min}$ at $80{ }^{\circ} \mathrm{C}$, from $80^{\circ} \mathrm{C}$ to $180{ }^{\circ} \mathrm{C}$ at $3{ }^{\circ} \mathrm{C} / \mathrm{min}, 10 \mathrm{~min}$ at $180{ }^{\circ} \mathrm{C}$, from $180{ }^{\circ} \mathrm{C}$ to $210^{\circ} \mathrm{C}$ at $5{ }^{\circ} \mathrm{C} / \mathrm{min}$; $5 \mathrm{~min}$ at $210{ }^{\circ} \mathrm{C}$. Carrier gas was He at $3 \mathrm{ml} / \mathrm{min}$. Detection of different aroma components was carried out by mass spectrometry, using a Clarus 560S mass spectrometer (Perkin Elmer) and EI + ionisation mode. A mass range of $m / z, 25-250$ was recorded each $0.5 \mathrm{~s}$, between 9 and $90 \mathrm{~min}$. Ion chromatograms and ion peaks were analysed using Turbomass GC/MS software, v. 5.4.2. Volatile compounds were identified comparing mass spectra of sample volatile compounds with mass spectra found in the NIST library, and by comparison of retention times and mass spectra of sample volatile compounds with those shown by standards; retention indices of volatile compounds were calculated. Quantification was performed from the calibration curves of the respective standards in solutions prepared in $12 \%$ ethanol at different concentrations. Those standard solutions, containing the volatile substances under study in the range of concentrations usually found for those compounds in young red wines, were prepared for the 19 volatile compounds analysed, dissolving the standards in $12 \%$ ethanol. The procedure was fully validated, following OIV procedures (OIV, 2010). Analyses were carried out in duplicate.

\subsection{Analysis of phenolic compounds}

Total anthocyanins and total condensed tannins were measured after three and nine months of bottling by visible-UV spectrophptometry, using an S-22 UV/Vis spectrophotometer (Boeco, Hamburg, Germany), following procedures previously described (Ribereau-Gayon \& Stonestreet, 1965; Ribereau-Gayon 6 Stonestreet, 1966). Analyses were carried out in duplicate.

\subsection{Determination of odour activity value $(O A V)$}

The specific contribution of each volatile compound on wine aroma was evaluated using the odour activity value (OAV). OAV is the ratio between the concentration of each volatile compound in wine and the perception threshold of that compound in wine (Belitz \& Grosch, 1999; Falque, Fernandez, \& Dubourdieu, 2001). It was considered that, for a given volatile compound, OAV $>1$ means a direct influence of that compound on wine aroma. Moreover, volatile components with OAV between 0.2 and 1 may contribute to wine aroma by synergistic effects (Belitz \& Grosch, 1999). The perception thresholds mentioned in Table 3 have been used for the calculation of OAV values.

\subsection{Statistical analysis}

The analyses of results using multifactor ANOVA and F-test techniques were performed using Statgraphics Centurion XIV statistical package (Statistical Graphics Corp., Warrenton, VA). Calculation of the linear correlation matrix was carried out using Microsoft Excel. Due to the high number of values, the analysis was carried out in a sequential way, regarding the interactions until the fourth order and successively rejecting those interactions that were not significant ( $\mathrm{p}>0.05$ ), pooling them within the experimental error. Some volatile components (e.g., 1-heptanol) were only detected three or nine months after bottling. In these cases, the factor length of storage in bottle was not considered for multifactor ANOVA. To evaluate the influence of total anthocyanins on aroma compounds, an additional set of multifactor ANOVA was performed, using the variable total anthocyanins as a random effect factor.

\section{Results and discussion}

\subsection{Physicochemical analysis of must and wines}

The physicochemical analysis of must showed results adequate for making young red wines, taking into account the reference values given by Appelation d'Origine Contrôlé Valdeorras: potential alcoholic degree, $12.70 ; \mathrm{pH}, 3.67$; total acidity, $6.20 \mathrm{~g} \mathrm{~L}^{\prime 1}$ (expressed as tartaric acid equivalents); tartaric acid, $4.00 \mathrm{gL}^{11}$; malic acid, $2.90 \mathrm{~g} \mathrm{~L}^{\prime \prime}$. The general analysis of wines revealed differences among samples, which are a consequence of the different technological factors used in the assays. The intervals for the different general parameters analysed in 32 wines were as follows: alcoholic degree, $12.37-13.16 \% \mathrm{v} / \mathrm{v} ; \mathrm{pH}, 3.61-3.84$; total acidity, 5.78-6.65 $\mathrm{gL}^{11}$ (expressed as tartaric acid equivalents); volatile acidity, 0.48-0.80 $\mathrm{g} \mathrm{L}^{\prime \prime}$ (expressed as acetic acid equivalents); dry extract, 33.1-33.5 g L"1; reducing sugars, 1.30-2.95 g L"1; total sulfur dioxide, 30-119 mg L"1; free sulfur dioxide, 5-36 mg L"1; malic acid, 0.10-0.16 $\mathrm{g} \mathrm{L"1}$. These data are typical of Mencia young red wines, as pointed out by Appelation d'Origine Controle Valdeorras, and the variation intervals for the different parameters are acceptable for the technological procedures assayed.

\subsection{Analysis of volatile and phenolic compounds}

Five mid-chain alcohols, 11 esters, two mid-chain fatty acids and benzaldehyde, that are fermentation-related compounds, were identified and quantified by GC-MS. Total anthocyanins and condensed tannins were determined by UV-visible spectrophotometry. Tables 1 and 2 show the values of those parameters in wines analysed after three and nine months of bottling, respectively. Four mid-chain alcohols (benzyl alcohol, 2-phenylethanol, 1-pentanol and 1-octanol) were present in all wines, but 1-heptanol was only detected in wines aged nine months in bottles. The most abundant was 2-phenylethanol; its concentration increased during storage in bottles. This effect is related to the hydrolysis of 2-phenylethyl acetate and of its glucoside precursors, as was previously demonstrated (Lopez, Ezpeleta, Sanchez, Cacho, \& Ferreira, 2004). The content of benzyl alcohol, 1-pentanol and 1-octanol decreased dramatically during the storage of wine in bottles; this fact has been described (Laurent, Henick-Kling, \& Acree, 1994), and it may be considered a consequence of the activity of residual lactic bacteria during storage in bottles.

The most abundant esters were ethyl lactate and diethyl succinate, whose concentrations, as well as the levels of isoamyl lactate, increased during storage in bottles, as a consequence of esters formation reactions (Herjavec \& Majdak, 2002). The levels of other ethyl esters (isovalerate, hexanoate, heptanoate, octanoate, pelargonate and pyruvate) and of isoamyl acetate decreased during storage in bottles, caused by their hydrolysis (Ramey \& Ough, 1980). The hydrolysis of ethyl hexanoate and ethyl octanoate was more intense in wines previously matured in oak barrels, which presented a lower $\mathrm{pH}$. Moreover, this effect may be caused, to some extent, by the absorption of some volatiles (e.g., ethyl octanoate) 
Table 1

Content of several volatile compounds, total anthocyanins and condensed tannins in wines three months after bottling. For key to wine codes, see Section 2.3 .

\begin{tabular}{|c|c|c|c|c|c|c|c|c|c|c|c|c|c|c|c|c|}
\hline \multirow[t]{2}{*}{ Compounds } & \multicolumn{16}{|l|}{ Wines } \\
\hline & $\mathrm{TA} 2 \mathrm{C} 3$ & TA2V3 & $\mathrm{TA} 3 \mathrm{C} 3$ & TA3V3 & TR2C3 & TR2V3 & TR3C3 & TR3V3 & FA2C3 & FA2V3 & FA3C3 & FA3V3 & $\mathrm{FR} 2 \mathrm{C} 3$ & FR2V3 & FR3C3 & FR3V3 \\
\hline \multicolumn{17}{|l|}{ Aromatic alcohols (ug/L) } \\
\hline Benzyl alcohol & 140 & 150 & 270 & 107 & 160 & 140 & 160 & 200 & 170 & 160 & 200 & 180 & 230 & 210 & 180 & 190 \\
\hline 2- Phenylethanol & 11,920 & 11,060 & 18,850 & 10,850 & 12,050 & 7,680 & 11,120 & 10,740 & 15,000 & 14,250 & 14,240 & 13,570 & 15,000 & 13,210 & 14,780 & 12,750 \\
\hline \multicolumn{17}{|l|}{ Mid-chain alcohols $(\mathrm{ug} / \mathrm{L})$} \\
\hline 1-Pentanol & 200 & 179 & 160 & 159 & 170 & 137 & 180 & 165 & 184 & 164 & 124 & 174 & 174 & 150 & 148 & 146 \\
\hline 1-Heptanol & nd & nd & nd & nd & nd & nd & nd & nd & nd & nd & nd & nd & nd & nd & nd & nd \\
\hline 1-Octanol & 40 & 34 & 43 & 30 & 30 & 25 & 35 & 30 & 35 & 41 & 37 & 31 & 40 & 26 & 28 & 29 \\
\hline \multicolumn{17}{|c|}{ Ethyl esters of fatty acids $(\mathrm{Ug} / \mathrm{L})$} \\
\hline Ethyl isovalerate & 7.2 & 7.2 & 3.3 & 9.3 & 5.8 & 7.4 & 4.8 & 9.3 & 7.0 & 7.1 & 3.8 & 8.5 & 7.6 & 10.8 & 6.9 & 7.5 \\
\hline Ethyl pelargonate & 2.4 & 2.0 & 5.4 & 1.9 & 1.8 & 2.0 & 1.9 & 1.8 & 2.0 & 2.1 & 2.1 & 2.0 & 3.0 & 1.5 & 2.2 & 2.6 \\
\hline Ethyl hexanoate & 502 & 424 & 391 & 427 & 384 & 310 & 327 & 410 & 451 & 435 & 426 & 419 & 441 & 322 & 340 & 354 \\
\hline Ethyl heptanoate & 5.2 & 4.1 & 4.4 & 4.5 & 3.6 & 3.1 & 3.0 & 4.1 & 4.6 & 4.3 & 4.1 & 3.9 & 5.0 & 2.5 & 2.7 & 2.7 \\
\hline Ethyl octanoate & 567 & 439 & 454 & 457 & 420 & 305 & 340 & 416 & 518 & 480 & 478 & 444 & 528 & 364 & 429 & 407 \\
\hline \multicolumn{17}{|c|}{ Esters of mid-chain alcohols $(\mathrm{ug} / \mathrm{L})$} \\
\hline Isoamyl acetate & 630 & 470 & 400 & 470 & 530 & 440 & 500 & 550 & 640 & 620 & 680 & 580 & 720 & 720 & 940 & 900 \\
\hline 2-Phenylethyl acetate & 30 & 30 & 20 & 20 & 20 & 30 & 30 & 20 & 50 & 60 & 30 & 30 & 30 & 30 & 40 & 40 \\
\hline Isoamyl lactate & 34 & 178 & 135 & 228 & 171 & 184 & 107 & 154 & 168 & 203 & 157 & 153 & 198 & 249 & 173 & 187 \\
\hline \multicolumn{17}{|c|}{ Ethyl esters of mid-chain organic acids $(\mathrm{Ug} / \mathrm{L})$} \\
\hline Ethyl pyruvate & 5.2 & 4.2 & 7.1 & 4.4 & 6.4 & 4.1 & 10.2 & 10.0 & 10.6 & 9.8 & 13.3 & 10.7 & 11.6 & 11.4 & 27.8 & 12.2 \\
\hline Ethyl lactate & 71,740 & 77,540 & 86,000 & 95,840 & 74,170 & 65,540 & 54,080 & 81,230 & 80,400 & 89,420 & 73,490 & 68,350 & 86,710 & 93,564 & 87,290 & 100,460 \\
\hline Diethyl succinate & 7139 & 7679 & 3030 & 7876 & 6489 & 5258 & 3966 & 6965 & 7768 & 8260 & 6706 & 5899 & 7768 & 8154 & 6965 & 7680 \\
\hline \multicolumn{17}{|c|}{ Other volatile compounds $(\mathrm{Ug} / \mathrm{L})$} \\
\hline Benzaldehyde & 196 & 145 & 172 & 118 & 117 & 90 & 97 & 63 & 195 & 164 & 98 & 87 & 175 & 68 & 68 & 58 \\
\hline Heptanoic acid & 3400 & 4600 & 2560 & 5300 & 6890 & 4001 & 4150 & 2890 & 6830 & 3110 & 2970 & 4360 & 6830 & 3060 & 2920 & 5410 \\
\hline Octanoic acid & 5500 & 5390 & 3550 & 6830 & 7040 & 3250 & 3430 & 6090 & 5160 & 6340 & 4720 & 5300 & 5160 & 5880 & 6390 & 4980 \\
\hline \multicolumn{17}{|c|}{ Phenolic compounds $(\mathrm{mg} / \mathrm{L})$} \\
\hline Total anthocyanins & 143 & 116 & 126 & 109 & 106 & 74 & 123 & 102 & 98 & 85 & 93 & 78 & 115 & 91 & 108 & 83 \\
\hline Condensed tannins & 2299 & 2196 & 2189 & 1817 & 1705 & 1667 & 1661 & 1652 & 2057 & 2185 & 2068 & 1633 & 1595 & 1380 & 1507 & 1336 \\
\hline
\end{tabular}

nd: not detected. 
Table 2

Content of several volatile compounds, total anthocyanins and condensed tannins in wines nine months after bottling. For key to wine codes, see Section 2.3 .

\begin{tabular}{|c|c|c|c|c|c|c|c|c|c|c|c|c|c|c|c|c|}
\hline \multirow[t]{2}{*}{ Compounds } & \multicolumn{16}{|l|}{ Wines } \\
\hline & $\mathrm{TA} 2 \mathrm{C} 9$ & TA2V9 & TA3C9 & TA3V9 & TR2C9 & TR2V9 & TR3C9 & TR3V9 & FA2C9 & FA2V9 & FA3C9 & FA3V9 & FR2C9 & FR2V9 & FR3C9 & FR3V9 \\
\hline \multicolumn{17}{|l|}{ Aromatic alcohols (ug/L) } \\
\hline Benzyl alcohol & 70 & 140 & 30 & 59 & 70 & 130 & 100 & 130 & 70 & 170 & 70 & 200 & 80 & 130 & 70 & 90 \\
\hline 2- Phenylethanol & 28,930 & 28,200 & 29,150 & 29,850 & 27,950 & 29,590 & 25,100 & 26,600 & 29,130 & 29,330 & 30,450 & 30,960 & 29,510 & 31,150 & 28,110 & 27,090 \\
\hline \multicolumn{17}{|l|}{ Mid-chain alcohols $(\mathrm{ug} / \mathrm{L})$} \\
\hline 1-Pentanol & 76 & 79 & 72 & 99 & 74 & 71 & 67 & 70 & 61 & 75 & 62 & 74 & 85 & 63 & 85 & 62 \\
\hline 1-Heptanol & 30 & 43 & 29 & 33 & 39 & 28 & 26 & 23 & 31 & 37 & 27 & 31 & 23 & 24 & 20 & 19 \\
\hline 1-Octanol & 18 & 20 & 18 & 20 & 18 & 21 & 13 & 14 & 18 & 23 & 17 & 25 & 12 & 17 & 11 & 12 \\
\hline \multicolumn{17}{|c|}{ Ethyl esters of fatty acids $(\mathrm{Ug} / \mathrm{L})$} \\
\hline Ethyl isovalerate & 0.5 & 1.3 & 0.3 & 0.1 & 0.7 & 1.3 & 0.1 & 0.1 & 0.6 & 1.3 & 0.3 & 1.2 & 0.2 & 0.8 & 0.1 & 0.6 \\
\hline Ethyl pelargonate & 2.0 & 0.4 & 0.2 & 0.3 & 0.3 & 0.4 & 0.2 & 0.2 & 0.3 & 0.4 & 0.2 & 0.5 & 0.2 & 0.3 & 0.2 & 0.2 \\
\hline Ethyl hexanoate & 59 & 45 & 45 & 56 & 33 & 42 & 34 & 26 & 55 & 53 & 46 & 64 & 21 & 40 & 21 & 24 \\
\hline Ethyl heptanoate & 0.1 & 0.2 & 0.1 & 0.1 & 0.2 & 0.1 & 0.1 & 0.2 & 0.1 & 0.2 & 0.1 & 0.2 & 0.2 & 0.1 & 0.1 & 0.1 \\
\hline Ethyl octanoate & 182 & 124 & 204 & 163 & 135 & 125 & 153 & 120 & 206 & 133 & 187 & 145 & 157 & 155 & 130 & 139 \\
\hline \multicolumn{17}{|c|}{ Esters of mid-chain alcohols $(\mathrm{ug} / \mathrm{L})$} \\
\hline Isoamyl acetate & 370 & 300 & 290 & 290 & 350 & 260 & 490 & 370 & 440 & 390 & 450 & 390 & 440 & 340 & 580 & 490 \\
\hline 2-Phenylethyl acetate & 20 & 10 & 20 & 20 & 20 & 20 & 30 & 70 & 30 & 20 & 40 & 30 & 40 & 20 & 50 & 80 \\
\hline Isoamyl lactate & 1077 & 772 & 916 & 1078 & 794 & 850 & 896 & 1060 & 764 & 749 & 764 & 1104 & 741 & 1005 & 1023 & 975 \\
\hline \multicolumn{17}{|c|}{ Ethyl esters of mid-chain organic acids $(\mathrm{Ug} / \mathrm{L})$} \\
\hline Ethyl pyruvate & 3.1 & 1.7 & 2.0 & 4.2 & 3.4 & 3.6 & 4.6 & 6.0 & 3.0 & 2.4 & 3.0 & 3.8 & 4.5 & 4.4 & 7.0 & 6 \\
\hline Ethyl lactate & 222,310 & 281,990 & 266,840 & 495,620 & 263,950 & 293,330 & 263,750 & 320,660 & 215,220 & 297,770 & 241,620 & 271,880 & 302,030 & 356,060 & 280,420 & 351,380 \\
\hline Diethyl succinate & 13,650 & 15,040 & 13,320 & 14,650 & 10,970 & 15,240 & 9,930 & 14,120 & 11,220 & 15,220 & 11,220 & 15,690 & 11,760 & 16,330 & 12,890 & 14,360 \\
\hline \multicolumn{17}{|c|}{ Other volatile compounds $(\mathrm{Ug} / \mathrm{L})$} \\
\hline Benzaldehyde & 51 & 37 & 45 & 82 & 39 & 27 & 19 & 14 & 70 & 41 & 70 & 33 & 39 & 23 & 34 & 17 \\
\hline Heptanoic acid & nd & nd & nd & 2.0 & 10 & nd & 10 & nd & nd & nd & nd & 10 & nd & 20 & 10 & 10 \\
\hline Octanoic acid & 8320 & 3090 & 7000 & 5780 & 6640 & 6730 & 6760 & 4170 & 7600 & 6170 & 5410 & 7450 & 7060 & 7680 & 4670 & 6430 \\
\hline \multicolumn{17}{|c|}{ Phenolic compounds $(\mathrm{mg} / \mathrm{L})$} \\
\hline Total anthocyanins & 81 & 72 & 74 & 62 & 63 & 46 & 66 & 61 & 66 & 59 & 64 & 52 & 60 & 48 & 59 & 45 \\
\hline Condensed tannins & 2090 & 1910 & 1990 & 1580 & 1550 & 1490 & 1510 & 1480 & 1870 & 1900 & 1880 & 1420 & 1450 & 1200 & 1370 & 1172 \\
\hline
\end{tabular}

nd: not detected. 
Table 3

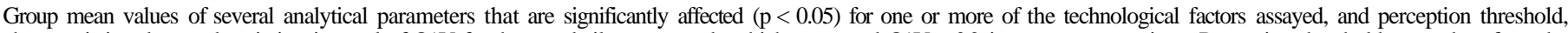

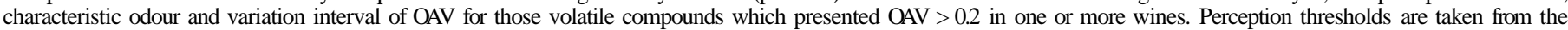

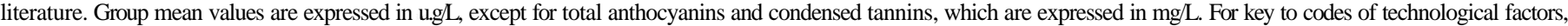
see Section 2.3

\begin{tabular}{|c|c|c|c|c|c|c|c|c|c|c|c|c|c|}
\hline \multirow[t]{3}{*}{ Compounds } & \multicolumn{10}{|c|}{ Technological factors } & \multirow{3}{*}{$\begin{array}{l}\text { Perception } \\
\text { threshold, red } \\
\text { wines }(\mathrm{ug} / \mathrm{L})\end{array}$} & \multirow{3}{*}{$\begin{array}{l}\text { Characteristic } \\
\text { odour }\end{array}$} & \multirow{3}{*}{$\begin{array}{l}\text { Variation } \\
\text { intervat. of } \mathrm{OAV}\end{array}$} \\
\hline & \multicolumn{2}{|c|}{$\begin{array}{l}\text { Type of } \\
\text { winemaking } \\
(\mathrm{Fl})\end{array}$} & \multicolumn{2}{|c|}{$\begin{array}{l}\text { Type of } \\
\text { container used } \\
\text { for maturation } \\
(\mathrm{F} 2)\end{array}$} & \multicolumn{2}{|c|}{$\begin{array}{l}\text { Length of } \\
\text { maturation } \\
\text { (F3) }\end{array}$} & \multicolumn{2}{|c|}{$\begin{array}{l}\text { Temperature of } \\
\text { storage in bottles } \\
\text { (F4) }\end{array}$} & \multicolumn{2}{|c|}{$\begin{array}{l}\text { Length of storage } \\
\text { in bottles (F5) }\end{array}$} & & & \\
\hline & $\mathrm{T}$ & $\mathrm{F}$ & A & $\mathrm{R}$ & 2 & 3 & $\mathrm{C}$ & V & 3 & 9 & & & \\
\hline Benzyl alcohol & & & & & & & & & 178 & 101 & $620^{3}$ & Fruity, blackberry ${ }^{3}$ & $0.03-0.30$ \\
\hline 2- Phenylethanol & 19,978 & 21,784 & 21,609 & 20,153 & & & & & 12,942 & 28,819 & $14,000^{\mathrm{b}}$ & Roses $^{\mathrm{b}}$ & $0.54-2.22$ \\
\hline 1-Pentanol & & & & & & & & & 163 & 73 & & & \\
\hline 1-Heptanol & 31 & 26 & & & 32 & 26 & & & 0.0 & 29 & & & \\
\hline 1-Octanol & & & & & & & & & 33 & 17 & & & \\
\hline Ethyl isovalerate & & & & & & & 3.1 & 4.6 & 7.1 & 0.6 & $3^{\mathrm{b}}$ & Fruity, lemon ${ }^{\mathrm{b}}$ & $0.03-3.60$ \\
\hline Ethyl hexanoate & & & 244 & 196 & & & & & 398 & 41 & $14^{\mathrm{b}}$ & Green apple $\mathrm{e}^{\mathrm{b}}$ & $4.20-100.40$ \\
\hline Ethyl heptanoate & & & 2.3 & 1.7 & & & & & 3.9 & 0.1 & & & \\
\hline Ethyl octanoate & & & 324 & 270 & & & 318 & 276 & 440 & 154 & $5^{\mathrm{b}}$ & Pineapple, pear $^{\mathrm{b}}$ & $24.00-113.40$ \\
\hline Ethyl pelargonate & & & & & & & & & 2.3 & 0.4 & & & \\
\hline Ethyl pyruvate & 5.0 & 8.9 & 5.5 & 8.4 & 5.6 & 8.4 & & & 9.9 & 4.0 & & & \\
\hline Ethyl lactate & & & & & & & 166,876 & 208,790 & 80,364 & 295,302 & $15.400^{3}$ & Butter $^{3}$ & $3.50-32.17$ \\
\hline Diethyl succinate & 9708 & 10,493 & & & 10,497 & 9704 & 9049 & 11,151 & 67 & 13,476 & $6000^{3}$ & Vinous $^{3}$ & $2.52-13.60$ \\
\hline Isoamyl acetate & 419 & 582 & 463 & 539 & 479 & 523 & 528 & 474 & 612 & 390 & $30^{\mathrm{c}}$ & Banana $^{c}$ & $8.66-31.33$ \\
\hline Isoamyl lactate & & & & & & & & & 167 & 910 & & & \\
\hline 2-Phenylethyl acetate & 26 & 39 & 29 & 36 & 29 & 36 & & & & & $250^{3}$ & Floral, roses ${ }^{3}$ & $0.08-0.32$ \\
\hline Benzaldehyde & & & 100 & 59 & 92 & 67 & 93 & 67 & 119 & 40 & & & \\
\hline Heptanoic acid & & & & & 4840 & 3820 & & & 4330 & 0.0 & $3000^{\mathrm{d}}$ & Oily, rancid ${ }^{\mathrm{d}}$ & $0.00-2.29$ \\
\hline Octanoic acid & & & & & & & & & 5313 & 6310 & $500^{\mathrm{b}}$ & Fatty acid, rancid ${ }^{\mathrm{b}}$ & $6.18-16.64$ \\
\hline Total anthocyanins & 89 & 76 & 86 & 79 & 83 & 82 & 90 & 74 & 104 & 61 & & & \\
\hline Condensed tannins & 1799 & 1626 & 1943 & 1483 & 1784 & 1642 & 1799 & 1626 & 1809 & 1616 & & & \\
\hline
\end{tabular}

a Noguerol-Pato et al. (2009)

berreira, Lopez, and Cacho (2000).

c Vilanova et al. (2012).

d Souid, Hassene, Palomo, Perez-Coello, and Ghorbel (2007).

by oak wood (Ramirez et al., 2001). In addition, wines obtained by prefermentative cryomaceration presented higher levels of 2phenylethyl acetate than others, caused by the development of cryophilic yeast (Charpentier \& Feuillat, 1998).

Among other aroma compounds, it should be pointed out that the levels of heptanoic acid and benzaldehyde decreased during storage in bottles, but the levels of octanoic acid increased simultaneously; the latter may be partially due to the hydrolysis of ethyl octanoate. Moreover, levels of benzaldehyde were higher in wines matured in stainless steel tanks.

Multifactor ANOVA indicates that the length of storage in bottles was the most important factor affecting the volatile composition of Mencia young red wines (Table 3 ). This factor significantly affected $(\mathrm{p}<0.05)$ most aroma compounds analysed, except 2phenylethyl acetate. On the other hand, other factors only affected significantly $(\mathrm{p}<0.05)$ eight, nine or ten of the 21 parameters under study. Three analytical parameters (isoamyl acetate, total anthocyanins and condensed tannins) changed significantly $(\mathrm{p}<0.05)$ in relation to the five factors studied. Four technological factors affected significantly $(\mathrm{p}<0.05)$ the levels of other three volatile compounds (ethyl pyruvate, diethyl succinate and benzaldehyde). 2-Phenylethyl acetate, the only analytical parameter that was not affected significantly by the length of storage in bottles, changed significantly $(\mathrm{p}<0.05)$ for other three technological factors. However, six parameters (benzyl alcohol, 1-pentanol, 1-octanol, ethyl pelargonate, isoamyl lactate and octanoic acid) only changed significantly $(\mathrm{p}<0.05)$ for one factor (length of storage in bottles). In the case of 1 -heptanol, its content was under the limit of detection after three months of maturation in bottle; thus, to study the effect of the other four factors, only data obtained after nine months of storage in bottles was taken into account.
Summarising, the technological practice that appeared to be most important for levels of fermentation-related aroma compounds in Mencia wines was the length of storage in bottles, that significantly affected $(\mathrm{p}<0.05) 18$ of the 19 aroma compounds analysed. Moreover, the only aroma compound that was not affected by this factor (2-phenylethyl acetate) was significantly affected $(\mathrm{p}<0.05)$ by factors F1, F2 and F3, related to winemaking and maturation procedures.

The study of the first, second and third order interactions among the five technological factors (F1, F2, F3, F4 and F5) and the 21 analytical parameters determined in wines is summarised in Supplementary Tables SI, S2 and S3, which show those interactions

Table 4

Linear correlation coefficients higher than 0.65 between the contents of phenolic compounds and fermentation-related aromas.

\begin{tabular}{lcc}
\hline Compounds & Total anthocyanins & Condensed tannins \\
\hline 2-Phenylethanol & -0.7847 & -0.2643 \\
1-Pentanol & 0.8724 & 0.3746 \\
1-Heptanol & -0.7537 & -0.1892 \\
1-Octanol & 0.8155 & 0.5567 \\
Ethyl isovalerate & 0.7015 & 0.1749 \\
Ethyl hexanoate & 0.8466 & 0.4261 \\
Ethyl heptanoate & 0.8501 & 0.4511 \\
Ethyl octanoate & 0.8572 & 0.4640 \\
Ethyl pelargonate & 0.7942 & 0.4434 \\
Ethyl lactate & -0.8045 & -0.4157 \\
Diethyl succinate & -0.8050 & -0.3427 \\
Isoamyl lactate & -0.8298 & -0.3860 \\
Benzaldehyde & 0.7952 & 0.6621 \\
Heptanoic acid & 0.7320 & 0.2309 \\
\hline
\end{tabular}


Table 5

Multifactor ANOVA for several aroma compounds, considering the five technological factors under study and the content of total anthocyanins as a random factor.

\begin{tabular}{|c|c|c|c|c|c|}
\hline Factors & & 1-Pentanol & Ethyl isovalerate & Ethyl octanoate & Ethyl lactate \\
\hline \multirow[t]{2}{*}{ Type of winemaking } & $\mathrm{F}$ & 0.08 & 20.12 & 9.31 & 2.96 \\
\hline & $\mathrm{P}$ & 0.7758 & 0.0012 & 0.0066 & 0.1000 \\
\hline \multirow{2}{*}{ Type of container used for maturation } & $\mathrm{F}$ & 0.29 & 17.66 & 17.66 & 0.20 \\
\hline & $\mathrm{P}$ & 0.5947 & 0.0018 & 0.0005 & 0.6585 \\
\hline \multirow[t]{2}{*}{ Length of maturation } & $\mathrm{F}$ & 1.84 & 3.24 & 0.80 & 1.40 \\
\hline & $\mathrm{P}$ & 0.1906 & 0.1021 & 0.3816 & 0.2493 \\
\hline \multirow[t]{2}{*}{ Temperature of storage in bottles } & $\mathrm{F}$ & 0.76 & 40.34 & 2.55 & 0.45 \\
\hline & $\mathrm{P}$ & 0.3943 & 0.0001 & 0.1266 & 0.5088 \\
\hline \multirow[t]{2}{*}{ Length of storage in bottles } & $\mathrm{F}$ & 48.26 & 0.02 & 83.96 & 14.08 \\
\hline & $\mathrm{P}$ & 0.0000 & 0.8939 & 0.0000 & 0.0012 \\
\hline \multirow[t]{2}{*}{ Total anthocyanins } & $\mathrm{F}$ & 5.29 & 17.55 & 6.17 & 5.90 \\
\hline & $\mathrm{P}$ & 0.0330 & 0.0019 & 0.0225 & 0.0242 \\
\hline
\end{tabular}

that were significant $(\mathrm{p}<0.05)$. The first order interaction between temperature of storage in bottles and length of storage in bottles (F4F5) was the most influential on the overall aroma profile; isoamyl acetate, 2-phenylethyl acetate and benzaldehyde being the aroma compounds most affected by first order interactions. The ethyl esters of fatty acids, as well as acetates, were also affected by the second order interaction between the previously mentioned factors and the length of maturation in containers (F3F4F5), as well as by the first order interactions F3F4 (length of maturation in containers and length of storage in bottles) and F2F5 (type of container used for maturation and temperature of storage in bottles). As was observed for first order interactions, isoamyl acetate, 2-phenylethyl acetate and benzaldehyde were the aroma compounds most affected by second and third order interactions. When single compounds were considered, it must be remarked that the contents of isoamyl acetate and 2-phenylethyl acetate were very sensitive to crossing effects between different factors and then, regarding the fact that variations due to main effects were small, the control of the content of these compounds by means of technological factors could be difficult. In the case of benzaldehyde, although several interactions were significant, they were negligible compared to the main effects.

Levels of total anthocyanins and condensed tannins were relatively low (Tables 1 and 2), as is expected in Mencia wines (Garcia-Falcon et al., 2007); the five technological factors had a significant effect $(\mathrm{p}<0.05)$ on the levels of these components (Table 3), as well as several first, second and third order interactions (Supplementary Tables SI, S2 and S3).

The study of the odour activity value (Table 3) showed that 11 volatile compounds presented OAV $>0.2$ for some of the wines under study. Moreover, nine volatile compounds presented $\mathrm{OAV}>1$ in some wines. Five volatile compounds formed during fermentation (ethyl lactate, octanoic acid, ethyl hexanoate, isoamyl acetate and ethyl octanoate) appear to be of importance to explain the aroma of Mencia wines, because they possessed OAV $>1$ in every wine. Ethyl octanoate presented the highest OAV among those five volatile compounds. Thus, it is likely that these wines may have a fruity aroma and, in some cases, a light floral aroma.

\subsection{Influence of phenolic composition on levels of aroma compounds}

The study of linear correlation between the levels of total anthocyanins and condensed tannins and the different aroma compounds under study was carried out, to study the possible influence of phenolic compounds on wine aroma. For this study, only those aroma compounds that presented linear correlation coefficients higher that 0.65 (in absolute value) with total anthocyanins or condensed tannins (Table 4) were selected. As can be seen, total anthocyanins present a high correlation with 14 different aroma compounds, but only benzaldehyde showed a high correlation with condensed tannins.

To evaluate the influence of total anthocyanins on aroma compounds, a new set of multifactor ANOVA was carried out, considering the content of total anthocyanins as a sixth random factor. These analyses have shown that aroma compounds may be classified into two groups. The first group includes those aroma compounds that are affected significantly $(\mathrm{p}<0.05)$ by the random factor total anthocyanins: 1-pentanol, ethyl isovalerate, ethyl octanoate and ethyl lactate. These data are shown in Table 5. The second group is made up of those aroma compounds that are not affected significantly by the random factor total anthocyanins, despite their high linear correlation with the content of total anthocyanins (Table 4): 2-phenylethanol, 1-heptanol, 1-octanol, ethyl pelargonate, ethyl heptanoate, isoamyl lactate, ethyl lactate and ethyl succinate.

Linear correlation between ethyl lactate and total anthocyanins is negative, but linear correlations between the other three volatile compounds of the first group mentioned above (ethyl isovalerate, ethyl octanoate and 1-pentanol) and total anthocyanins are positive. Thus, it appears that total anthocyanins may present a protective effect on those last three aroma compounds, probably due to the retention of these three molecules by coloured colloids present in red wines. As a consequence, the aromatic expression of Mencia wines could be affected to some extent by the content of total anthocyanins. Moreover, ethyl lactate and ethyl octanoate presented OAV $>1$ in all the studied wines, and OAV values were remarkable high for ethyl octanoate (Table 3). Thus, we may conclude that Mencia young red wines with a high content of total anthocyanins, that can be easily measured in the laboratory, are likely to contain higher levels of ethyl octanaoate (with a characteristic aroma of pineapple and pear) and lower levels of ethyl lactate (with a characteristic aroma of butter) than Mencia young red wines with low colour. These results may be of importance for winemakers, because the contribution of anthocyanins to taste quality scores of red wines is well established (Sommers \& Evans, 1974), and taste quality scores are the primary marketing information for high-priced wines in many wine markets, like California (Eyler, 1999).

\section{Conclusions}

Several conclusions can be drawn from the assays. First, the length of storage in bottle was the most significant technological procedure on the levels of fermentation-related aromas in young red Mencia wines. Moreover, when first order interactions among the five technological factors assayed were considered, the most influential factors on the aroma profile of those Mencia wines were those factors related to storage in bottles: length of storage and 
temperature of storage. Second, five aroma compounds presented OAV > 1 (ethyl hexanoate, ethyl octanoate, ethyl lactate, isoamyl acetate and octanoic acid) in young red Mencia wines, and they can explain characteristic Mencia fruity aroma. Finally, the content of total anthocyanins in young red Mencia wines affects significantly the levels of four fermentation-related aromas, including ethyl octanoate and ethyl lactate, which presented OAV $>1$.

\section{Acknowledgements}

Authors acknowledge to Jesus Nazareno Cooperative Cellar (El Barco de Valdeorras, Orense, Spain) by its valuable help and to Dr. J. Andres for editing the manuscript.

\section{Appendix A. Supplementary data}

Supplementary data associated with this article can be found, in the online version, at http://dx.doi.Org/10.1016/j.foodchem.2013. 10.056 .

\section{References}

Alvarez, I., Aleixandre, J. L., Garcia, M. J., \& Lizama, V. (2005). Impact of prefermentative maceration on the phenolic and volatile compounds in Monastrell red wines. Analytica Chimica Acta, 563,109-115.

Belitz, H. D., \& Grosch, W. (1999). Food chemistry (2nd ed.). Berlin: Springer-Verlag.

Calleja, A, \& Falque, E. (2005). Volatile composition of Mencia wines. Food Chemistry, 90, 357-363.

Callejon, R. M., Clavijo, A., Ortigueira, P., Troncoso, A M., Paneque, P., \& Morales, M. L. (2010). Volatile and sensory profile of organic red wines produced by different selected autochthonous and commercial Saccharomyces cerevisiae strains. Analytica Chimica Acta, 660, 68-75.

Chalier, P., Angot, B., Delteil, D., Thierry, D., \& Gunata, Z. (2007). Intercations between aroma components and whole mannoprotein isolated from Saccharomyces cerevisiae strains. Food Chemistry, 100, 22-30.

Charpentier, C, \& Feuillat, M. (1998). Metabolisme des levures cryotolérants: application à la maceration prèfermentaire à froid du Pinot Noir en Bourgogne. Revue Francaise d'Oenologie, 170, 36-37.

Chatonnet, P., Boidron, J. N., \& Pons, M. (1990). Elevage des vins rouges en fûts de chene: evolutión de certains composes volatils et de leur impact aromatique. Science des Aliments, 10, 565-587.

Eyler, R. C. (1999). The international competitiveness of the California wine industry. Rohnert Park, CA: Sonoma State University.

Falque, E., Fernandez, E., \& Dubourdieu, D. (2001). Differentiation of white wines by their aromatic index. Talanta, 54, 271-281

Ferreira, V., Lopez, R., \& Cacho, J. F. (2000). Quantitative determination of the odorants of young red wines from different grape varieties. Journal of the Science of Food and Agriculture, 80,1659-1667.

Garcia-Falcon, M. S., Perez-Lamela, C, Martinez-Carballo, E., \& Simal-Gandara, J. (2007). Determination of phenolic compounds in wines: influence of bottle storage of young red wines on their evolution. Food Chemistry, 105, 248-259.
Herjavec, S., \& Majdak, A. (2002). The influence of maceration on the composition of some volatile compounds and sensory properties of Traminer wines. Agriculturae Conspectus Scientificus, 67,11-17.

Laurent, M. H., Henick-Kling, T., \& Acree, T. E. (1994). Changes in the aroma and olor of Chardonnay wine due to malolactic fermentation. Die Weinwissenschaft, 49, $3-10$.

Lopez, R, Ezpeleta, E., Sanchez, I., Cacho, J. F, \& Ferreira, V. (2004). Analysis of the aroma intensities of volatile compounds released from mild acid hydrolysates of odorless precursors extracted from Tempranillo and Grenache grapes using gas chromatography-olfactometry. Food Chemistry, 88, 95-103.

Lubbers, S., Charpentier, C, Feuillat, M., \& Voilley, A. (1994). Influence of yeast walls on the behavior of aroma compounds in a model wine. American Journal of Enology and Viticulture, 45, 29-33.

Murat, M. L., Tominaga, T., Saucier, C, Glories, Y., \& Dubourdieu, D. (2003). Effect of anthocyanins on stability of a key odorous compound, 3-mercaptohexan-1-ol, in Bordeaux rose wines. American Journal of Enology and Viticulture, 54,135-138.

Noguerol-Pato, R., Gonzalez-Barreiro, C, Cancho-Grande, B., \& Simal-Gandara, J. (2009). Quantitative determination and characterisation of the main odorants of Mencia monovarietal red wines. Food Chemistry, 117, 473-484.

OIV (2010). Compendium of international methods of wine and must analysis. Paris: International Organisation of Vine and Wine

Ortega-Heras, M., Perez-Magarifio, S., \& Gonzalez-Sanjose, M. L. (2012). Comparative study of the use of maceration enzymes and cold prefermentative maceration on phenolic and anthocyanic composition and colour of a Mencia red wine. LWT-Food Science and Technology, 48, 1-8.

Perestrelo, R., Fernandes, A, Alburquerque, F. F., Marques, J. C, \& Camara, J. S. (2006). Analytical characterization of the aroma of Tinta Negra Mole red wine: identification of the main odorants compounds. Analytica Chimica Acta, 563, 154-164.

Prieto, N., Rodriguez-Mendez, M. L., Leardi, R., Oliveri, P., Hernando-Esquivel, D., Iñiguez-Crespo, M., \& De Saja, J. A. (2012). Application of multi-way analysis to UV-visible spectroscopy, gas chromatography and electronic nose data for wine ageing evaluation. Analytica Chimica Acta, 719, 43-51.

Puech, C, Vidal, S., Pegaz, J. F., Riou, C, \& Vuchot, P. (2006). Influence des conditions de conservation des vins en bouteille sur leur évolution. Revue des Oenologes et des Techniques Vitivinicoles et Oenologiques, 121, 13-16.

Ramey, D. D., \& Ough, C. S. (1980). Volatile ester hydrolysis or formation during storage of model solutions and wines. Journal of Agricultural and Food Chemistry, $28,928-934$

Ramirez, G., Lubbers, S., Charpentier, C, Feuillat, M., Voilley, A., \& Chassagne, D. (2001). Aroma compound sorption by oak wood in a model wine. Journal of Agricultural and Food Chemistry, 49, 3893-3897.

Rapp, A., \& Mandery, H. (1986). Wine aroma. Experientia, 42, 873-884.

Ribereau-Gayon, P., \& Stonestreet, E. (1965). Le dosage des anthocyanes dans le vin rouge. Bulletin de la Societé Chimique de France, 1965, 2649-2652.

Ribereau-Gayon, P., \& Stonestreet, E. (1966). Dosage des tannins du vin rouge et détermination de leur structure. Chimie Analitique, 48,188-196.

Sommers, T. C, \& Evans, M. E. (1974). Wine quality: correlations with color density and anthocyanin equilibria in a group of young red wines. Journal of the Science of Food and Agriculture, 25,1369-1379.

Souid, I., Hassene, Z., Palomo, E. S., Perez-Coello, M. S., \& Ghorbel, A. (2007). Varietal aroma compounds of Vitis vinifera $\mathrm{L}$ cv Khamri grown in Tunisia. Journal of Food Quality, 30, 718-730.

Vilanova, M., Campo, E., Escudero, A., Graña, M., Masa, A., \& Cacho, J. (2012). Volatile composition and sensory properties of Vitis vinifera red cultivars from North West Spain: correlation between sensory and instrumental analysis. Analytica Chimica Acta, 720,104-111. 